\title{
Trauma and Massive Blood Transfusions
}

\author{
Marc P. Steurer · Michael T. Ganter
}

Published online: 1 May 2014

(C) Springer Science + Business Media New York 2014

\begin{abstract}
Massive hemorrhage in trauma requires a multitude of interventions in order to obtain the best possible outcome. The resuscitative approach of the actively bleeding trauma patient has evolved dramatically over the past decade. Modern strategies deploy an earlier, more aggressive and holistic approach on both the diagnostic and therapeutic side. This review addresses the physiology of hemorrhage and traumatic coagulopathy, current treatment options, including damage control resuscitation, individualized and tailored coagulation management with the application of point-of-care testing, and the use of blood and procoagulant products.
\end{abstract}

Keywords Trauma - Massive transfusion (MT) - Acute traumatic coagulopathy (ATC) - Transfusion ratio · Goal directed coagulation management - Damage control resuscitation (DCR)

\section{Introduction}

Trauma remains one of the leading causes of premature mortality and disability around the globe, representing a substantial burden on our society [1,2]. Despite numerous advancements in the field of trauma care, exsanguination is

M. P. Steurer $(\bowtie)$

Department of Anesthesia and Perioperative Care, San Francisco

General Hospital, University of California San Francisco, 1001

Potrero Ave., Room 3C-38, San Francisco, CA 94110, USA

e-mail: steurerm@anesthesia.ucsf.edu

\section{T. Ganter}

Institute of Anesthesiology and Pain Medicine, Kantonsspital Winterthur, Brauerstrasse 15, 8401 Winterthur, Switzerland

e-mail: michael.ganter@ksw.ch still the major contributor in over half the cases of traumaassociated deaths within the first $48 \mathrm{~h}$ of hospital admission $[3,4]$.

Trauma, massive bleeding, hemorrhagic shock and consecutive resuscitation with fluids and transfusion all massively activate the innate immune system, endothelium and coagulation system [5, 6]. The nomenclature surrounding the resulting coagulopathy is not entirely uniform. Frith and Brohi describe the entity in a clean fashion: trauma and shock by itself leads to an endogenous acute traumatic coagulopathy (ATC) $[7 \cdot \bullet]$. The mechanisms that lead to ATC can be summarized as follows: systemic anticoagulation and hyperfibrinolysis thru activation of Protein C, which in return leads to the inactivation of Factors V and VIII (coagulopathy) and consumption of PAI-1 (hyperfibrinolysis). In addition, the activation and dysfunction of platelets plus the endothelium also play an important role in the evolving coagulopathy. If the trauma patient cannot be stabilized at this stage, other factors like blood loss, dilution (plus the side effects of replacement fluids), consumption, hypocalcemia, hypothermia and acidosis are worsening the coagulation status even further, and the overall product is then called the multifactorial trauma induced coagulopathy (TIC) [8].

Cohen et al. [9•] were able to demonstrate in a prospective cohort that the early, endogenous ATC with its activation of the Protein $\mathrm{C}$ pathway is clearly linked to a higher mortality and incidence of organ failure and transfusion requirements. Along with the discovery and awareness for TIC, concepts to avoid and aggressively correct it early have evolved. Since data from the CRASH2 trial became available, trauma patients are now being treated with anti-fibrinolytics in the very beginning, since this intervention has been shown to clearly reduce all-cause mortality [10]. Observations from the armed conflicts in the 
Middle East have lead to a shift towards a hemostatic resuscitation concept in the U.S. that includes judicious use of crystalloids, early administration of blood products, a matched ratio of $\mathrm{PRBC} / \mathrm{FFP} /$ platelets, and lower target blood pressures [11]. Finally in Europe, specific goaldirected therapy of TIC with coagulation factor concentrates guided by point-of-care coagulation monitoring has become more and more the accepted standard of care $[12 \bullet \bullet]$.

\section{Pathogenesis of Bleeding in Trauma}

The presence of post traumatic coagulopathy has been recognized for a long time and described since the late 1960s [13, 14]. But the concept of the coagulopathy being an independent risk factor for significant morbidity and mortality only evolved in the 1990s. MacLeod was one of the first to properly describe the effect of a disturbed coagulation system on the outcome of a trauma patient [15]. She reviewed the prospectively collected data of 7,638 patients that presented to a Level I trauma center and was able to show that an abnormal prothrombin time (PT) and partial thromboplastin time (PTT) was associated with an adjusted odds ratio for death of 1.35 and 4.26, respectively.

Over time the understanding and nomenclature of the post trauma coagulopathy have become better studied and defined. The acute traumatic coagulopathy (ATC) is an endogenous process that develops quickly and early after the initial traumatic impact on the body. Two studies were able to clearly demonstrate that over $50 \%$ of trauma victims already have an abnormal coagulation status on the scene before any fluids were administered as measured by both classic coagulation parameters and thrombelastography $[16,17]$. The main driving forces behind the ATC are tissue damage and systemic hypoperfusion, although the exact contribution and role of each remains to be further specified $[18,19]$. Adding the "lethal triad of trauma", i.e., hypothermia, acidosis and hemodilution to the endogenous ATC results in the more comprehensive trauma induced coagulopathy (TIC), as described by Brohi [7••]. In contrast to the ATC, the TIC is at least to a certain degree an iatrogenic phenomenon, and, as such, in parts avoidable. Retrospective studies by both Wafasaide and Hubetamann were able to demonstrate that hypothermia and/or administration of large volumes of crystalloids worsened the patients' coagulopathy, increased the number of transfused blood products and had negative effects on outcomes such as sepsis and organ failure [20,21].

Present understanding of the hemostasis encompasses the balance among procoagulant and anticoagulant factors, platelets, endothelium and the fibrinolytic cascade (Fig. 1).

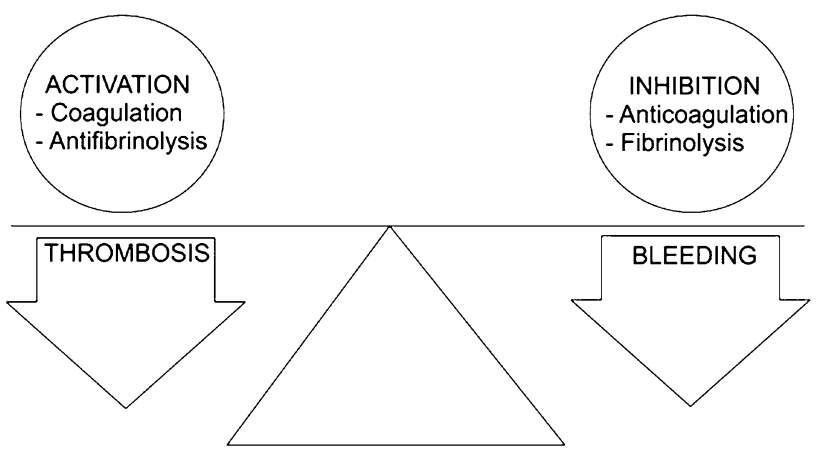

Fig. 1 Normal blood coagulation. The goal of every intervention in the coagulation system should be to restore normal blood coagulation, i.e., homeostasis, so that forces activating and inhibiting coagulation are in balance

The activation of the Protein $\mathrm{C}$ pathway and the depletion of Protein C itself appear to be a key trigger in the development of ATC [8]. The activated Protein C (APC) acts as a functional anticoagulant and inactivates Factors $\mathrm{V}$ and VIII. The prospectively randomized CRASH-2 trial has clearly highlighted the role of hyperfibrinolysis in ATC [10].A recent study by Raza et al. [22] found that over $60 \%$ of trauma patients have evidence of at least a moderate degree of hyperfibrinolysis. Furthermore, the magnitude of hyperfibrinolysis correlated with poor clinical outcome. The role of the platelets in ATC remains indistinct. On one side, the measureable decrease in number and function of the platelets seems questionably relevant at best [23]. On the other side, if a platelet deficit was present, there seemed to be an association with worse outcome. Additionally, there is data emerging that increased and earlier transfusion of platelets leads to improved early and late survival in trauma patients [24]. All of this leads to the conclusion that the functional platelet mass plays a crucial role in the ATC and is of distinct importance early on. The vascular endothelium and its glycocalyx also play a very important role in the development of ATC [25].

\section{Basic Principles in the Treatment of Massive Bleeding}

Depending on the amount of blood lost, hemorrhage can be classified into four stages (Table 1). Severely bleeding patients will usually require massive transfusion (MT). The concept of MT has been mostly arbitrarily defined and evolved over the past few decades. The different definitions of MT can lead to comparisons and conclusions that may be erroneous based on the different entities that are compared. One of the early definitions of MT circumscribed the application of ten or more units of blood within the first $24 \mathrm{~h}$ as criteria. Other definitions for MT are transfusion of $>4 \mathrm{RBC}$ units in $1 \mathrm{~h}$ or replacement of $>50 \%$ of total 
Table 1 Hemorrhage Classification (per ATLS 9th edition)

\begin{tabular}{lllll}
\hline Estimated blood loss ${ }^{\text {a }}$ based on patient's initial presentation & & & \\
\hline & Class I & Class II & Class III & Class IV \\
\hline Blood loss (mL) & Up to 750 & $750-1,500$ & $1,500-2,000$ & $>2,000$ \\
Blood loss (\% blood volume) & Up to $15 \%$ & $15-30 \%$ & $30-10 \%$ & $>40 \%$ \\
Pulse rate (BPM) & $<100$ & $100-120$ & $120-140$ & $>140$ \\
Systolic blood pressure & Normal & Normal & Decreased & Decreased \\
Pulse pressure & Normal or increased & Decreased & Decreased & Decreased \\
Respiratory rate & $14-20$ & $20-30$ & $30-40$ & $>35$ \\
Urine output (ml/hr) & $>30$ & $20-30$ & $5-15$ & Negligible \\
CNS/mental status & Slightly anxious & Mildly anxious & Anxious, confused & Confused, lethargic \\
Initial fluid replacement & Crystalloid & Crystalloid & Crystalloid and blood & Crystalloid and blood \\
\hline
\end{tabular}

${ }^{\text {a }}$ For a $70-\mathrm{kg}$ man

From Advanced Trauma Life Support (ATLS) manual, 9th edition, with permission from the American College of Surgeons

blood volume by blood products within $3 \mathrm{~h}$ [26]. It can easily be appreciated that not all patients fulfilling this definition can be compared with regards to physiology and intervention.

A key component for severely hemorrhaging patients is the development of a standardized institutional massive transfusion protocol (MTP). It facilitates communication, minimizes the response time and helps avoid errors. When implementing and using them, MTPs have been able to demonstrate better patient survival with lower rates of organ failure and other complications [27]. Even though, the data in those trials is usually not prospectively randomized, they do not demonstrate any harm, and it seems reasonable to have a well orchestrated response in place for a given facility.

When taking care of trauma patients with a massive bleeding event, it is of paramount importance to stratify the goals for resuscitation. For each patient, they should be divided in circulating blood volume, oxygen carrying capacity plus delivery and coagulation status (Table 2). The breakdown in these three individual targets helps the clinician to address each with the appropriate measures.

\section{Circulating Blood Volume}

In the early resuscitation phase, before the source of hemorrhage is controlled, there has been a clear shift over the past $10-15$ years towards a damage control resuscitation (DCR) concept. Data from the military conflicts in the Middle East and a better understanding of traumatic coagulopathy have led to this movement. On the resuscitation side, DCR consists of permissive hypotension and hemostatic resuscitation [28]. Permissive hypotension accepts lower blood pressures as long as the patient is actively hemorrhaging, and by doing so minimizing one of
Table 2 Pillars of resuscitation for massive hemorrhage

(1) Circulating blood volume

(a) Management before surgical control of bleeding: Damage control resuscitation, permissive hypotension, hemostatic resuscitation

(b) Management after surgical control of bleeding:

Restoration of normal circulating blood volume and cardiac output

(2) Oxygen carrying capacity and delivery

(a) Adequate volemia

(b) Adequate oxygen carrier (hemoglobin)

(c) Adequate oxygenation

(3) Blood and coagulation status

(a) Massive transfusion protocol

(b) Massive transfusion algorithm

(c) Preconditions normalization ( $\mathrm{pH}$, calcium, temperature)

(d) Anti-fibrinolytics

(e) Factor concentrates (fibrinogen, PCC, F XIII, rF VIIa, other), cryoprecipitate, FFP

(f) Platelet concentrates

(g) Protamine, desmopressin

$P C C$ prothrombin complex concentrate, $F$ XIII coagulation factor XIII, $r F$ VII recombinant activated coagulation factor VII, $F F P$ fresh frozen plasma

the driving forces behind the ongoing blood loss. Usually resuscitative efforts are guided such that a radial pulse is just palpable. One has to be cautious in the setting of TBI where there is a possible conundrum between systemic blood loss and cerebral perfusion pressure. DCR helps avoid and counteract acidosis, hypothermia and coagulopathy. As a result, it can help patients achieving critical hemostasis before surgical control. During the late phase of resuscitation, once hemostasis has occurred, the paradigm 
then shifts towards a normalization of circulatory parameters. Restoration of adequate circulating blood volume and cardiac output enables the perfusion of all vital tissues and normalization of the physiologic state. During this stage more advanced and invasive monitoring becomes essential [29].

\section{Oxygen Carrying Capacity and Delivery}

Restoring both, the oxygen carrying capacity (OCC) and oxygen delivery $\left(\mathrm{DO}_{2}\right)$ are key goals of resuscitation in trauma, especially once hemostasis is achieved. Re-establishing normal levels of $\mathrm{DO}_{2}$ will enable the patient to normalize his organ functions and metabolic status. A closer look at the equation circumscribing the oxygen delivery helps understand the entity better:

$\mathrm{DO}_{2}=\mathrm{CO} \times \mathrm{CaO}_{2}$

$\mathrm{CaO}_{2}=\left(\mathrm{Hb} \times \mathrm{SpO}_{2} \times \mathrm{k}_{1}+\mathrm{PaO}_{2} \times \mathrm{k}_{2}\right)$,

where $\mathrm{CO}$ is the cardiac output, $\mathrm{CaO}_{2}$ the oxygen content of the arterial blood, $\mathrm{Hb}$ the hemoglobin, $\mathrm{SpO}_{2}$ the arterial $\mathrm{Hb}$ saturation, $\mathrm{PaO}_{2}$ the arterial oxygen partial pressure, $\mathrm{k}_{1}$ the oxygen combining capacity of $\mathrm{Hb}$ (also sometimes referred to as Hüfner's constant) and $k_{2}$ the solubility coefficient of oxygen at body temperature. The exact value for $\mathrm{k}_{1}$ is somewhat controversial but in theory each gram of $\mathrm{Hb}$ binds $1.39 \mathrm{ml}$ of oxygen. In practice, however, the existence of abnormal hemoglobins reduces this capacity to a value of $1.31 \mathrm{ml} \mathrm{g}^{-1}$. $\mathrm{K}_{2}$ is equal to $0.003 \mathrm{ml}$ of oxygen per deciliter of plasma and per millimeter mercury pressure $(\mathrm{mmHg})$. While $\mathrm{k}_{2}$ is relatively small and does not contribute much to the $\mathrm{CaO}_{2}$ under normal circumstances, it can become vital in the hemorrhaging patient, where a significant increase in oxygen partial pressure $\left(\mathrm{FiO}_{2}=1.0\right)$ can make up for some amount of $\mathrm{Hb}$ lost. The formula for oxygen delivery illustrates clearly the need for an adequate $\mathrm{CO}$, which is usually achieved by restoring the circulating blood volume once hemostatic control is achieved. In most trauma patient's inotropic support for the heart is not needed to further support CO.

A bleeding patient's $\mathrm{Hb}$ is restored with the transfusion of red blood cells (RBC). Exact transfusion triggers for $\mathrm{Hb}$ remain debated, individual and controversial for most settings. In one of the more thorough analyses, the Eastern Association for the Surgery of Trauma (EAST) and the Society of Critical Care Medicine (SCCM) have assessed all scientific evidence that was available from 1980 thru 2008 and came up with the following indications for RBC transfusion in critically ill trauma patients [30]:

Hemorrhagic shock.

Hemodynamic instability and/or inadequate $\mathrm{DO}_{2}$ with acute hemorrhage.
A pure $\mathrm{Hb}$ based trigger of $<7 \mathrm{~g} / \mathrm{dl}$ in resuscitated trauma patients.

Use co-triggers such as intravascular volume status, evidence of hypoperfusion, duration and extent of anemia, cardiopulmonary parameters.

\section{Coagulation Status}

The correction and support of the coagulation system in a massively bleeding patient is potentially the most complex and challenging aspect during the resuscitation of a massively bleeding trauma patient. At the same time it appears to be an area where avoiding mistakes and doing the right things can lead to improved patient outcome. For this reason, we are addressing the topic of coagulopathy and its management in more detail.

\section{Assessment of the Hemostatic System}

To best assess and judge the coagulation system, information regarding the following four pillars of perioperative coagulation monitoring are being collected and require combined analysis.

\section{Patients' Medical History}

The focused patients' medical history is crucial in the judgment of the individual bleeding risk. To raise sensitivity and specificity, a given patients' medical history including current medication should be carried out with particular questionnaires. This standardized assessment has been shown to be superior to pre-operative routine lab coagulation studies alone [31]. Accordingly, many national societies have published recommendations on standardized pre-operative assessment of hemostasis, like the questionnaire designed by the Austrian society of anesthesiology, resuscitation and intensive care medicine [32].

\section{Clinical Presentation}

The clinical presentation of abnormal hemostasis (e.g., certain phenotypes of bleeding or thrombosis) is critical for the differential diagnosis and gives valuable information on possible etiologies of the underlying coagulation disorder. On the other hand, it is quintessential that abnormal lab coagulation studies are always correlated with the current clinical presentation, before any hemostatic therapy is being initiated. Without any clinically relevant diffuse bleeding (e.g., "dry" surgical area) no pro-coagulant therapy should be initiated, as it may increase the risk of 
thrombotic events. Instead, the patient must be closely observed and re-assessed.

When a patient is bleeding, the question often arises whether the cause of bleeding is "surgical" or "non-surgical". Clinically, the latter is usually characterized by a more diffuse pattern. In addition, advanced coagulation monitoring can help to distinguish both types of bleeders. If a "surgical" bleeding is indeed present, the patient must undergo surgical re-exploration and hemostasis. A diffuse, microvascular, "non-surgical" hemorrhage, however, requires quick, individualized and goal-directed pro-coagulant interventions.

\section{Standard Lab Coagulation Tests}

Standard lab coagulation tests include PT/INR, aPTT and platelet count. Depending on local circumstances, other lab values such as fibrinogen concentration, factor XIII (FXIII) and thrombin time may be part of a routine lab coagulation battery.

Standard lab coagulation tests play a central role in the initial diagnostic steps of patients with deranged hemostasis. However, when isolated in use, the standard lab parameters come with significant limitations regarding the detection of extent and origin of a given patient's coagulopathy. These tests allow only the determination of certain questions and are of restricted value [33].

Patients presenting with complex hemostatic disorders require more and in-depth coagulation studies. Discussion of these advanced lab coagulation tests is beyond the scope of this article.

\section{Bedside (point-of-care) Coagulation Tests}

There are several methods available to analyze blood coagulation at the bedside. Basically, the methods can be divided into the following categories [33, 34]:

Platelet function monitoring. To assess primary hemostasis, platelet count (quantity) and platelet function (quality) have to be measured. Several bedside tests are available to assess platelet function (e.g., PFA-100 and modified platelet aggregometry) $[35,36]$.

Analysis of secondary (plasmatic) hemostasis. These bedside tests are used to monitor anticoagulant therapy. Examples are measurement of activated clotting time (ACT), whole blood PT/INR, and whole blood aPTT [34]. Global, whole blood coagulation assessment. Viscoelastic coagulation monitoring devices like TEG/ROTEM and Sonoclot assess the whole coagulation process from initial thrombin generation to maximum clot formation up to clot lysis [33, 37•].

Bedside coagulation tests, especially viscoelastic tests can help tailor a patient's coagulation management and may reduce unnecessary administration of pro-coagulant substances (transfusions and coagulation factor concentrates) and enable the clinicians to distinguish between a surgical cause of bleeding and coagulopathy. These tests may also be a tool to avoid interventional delay as well as reduce surgical procedures because of bleeding. Ultimately, they may reduce mortality.

\section{Treatment of Coagulopathy}

With the information from the aforementioned four pillars of perioperative coagulation monitoring, bleeding patients can be treated individually with a goal-directed approach according to predefined algorithms. Evidence-based guidelines, like the one from the "Advanced Bleeding Care in Trauma" (ABC-T) [12*. ] or the European Society of Anaesthesiology [38] are helpful in developing locally adapted treatment algorithms.

It must be emphasized that pro-coagulant therapy should always be applied with caution. A deficient coagulation system should never be excessively corrected; otherwise, there is a serious risk for thrombo-embolic adverse events (Fig. 1). Therapy should be titrated carefully, using point-of-care testing and stopped if the bleeding is no longer clinically relevant.

\section{Goal-directed Coagulation Management, Algorithms}

A specific, goal-directed coagulation management in combination with clearly defined algorithms can lead to decreased transfusion needs, diminished costs and a better outcome [39-41]. Therefore, transfusion algorithms have recently been introduced in various clinics (e.g., a specific algorithm at a Swiss [42] or Austrian [43•] level I trauma center). Algorithms consider the physiology and pathophysiology of the developing coagulopathy in massively bleeding patients and are based on the four, above mentioned pillars of perioperative coagulation management. They serve as clearly structured guidelines for a specific and individualized coagulation therapy [42].

\section{Preconditions}

The coagulation system is a complex and delicate network of multiple interacting enzymatic processes. Several preconditions are mandatory for a normally functioning hemostatic process.

\section{Acid Base Status}

The activity of most coagulation proteins depends on a physiological $\mathrm{pH}$. For example, a drop in $\mathrm{pH}$ to 7.1 lowers their activity to $50 \%$. Besides a compromised clotting process, acidosis further accelerates fibrinogen consumption and 
impairs platelet function. Interestingly, correction of an established acidosis with, e.g., bicarbonate that returns arterial $\mathrm{pH}$ to 7.4 does not restore normal coagulation in a time-sensitive way [44]. Current guidelines, therefore advise, to primarily avoid acidosis and maintain an arterial $\mathrm{pH}>7.2$ [45].

\section{Calcium}

Calcium is an essential co-factor for normal coagulation within a normal range of free, ionized calcium between 1.1 and $1.3 \mathrm{mmol} / \mathrm{L}$. Massive transfusion may reduce ionized calcium via dilution, consumption and citrate calcium complexing. To avoid impaired hemostasis and hemodynamics, ionized calcium should be kept above $0.9 \mathrm{mmol} / \mathrm{L}$ [45].

\section{Body Temperature}

The body temperature itself also has significant influences on the hemostasis. According to van't Hoff and Arrhenius, each single drop of $1{ }^{\circ} \mathrm{C}$ reduces the activity of coagulation proteases by $4-10 \%$ [46]. Besides impaired enzymatic reactions, hypothermia also impairs platelet function and increases fibrinolytic activity. Therefore, every effort has to be made to keep bleeding patients normothermic.

\section{Coagulation Factor Concentrates}

Several coagulation factor concentrates are commercially available today and can be used for specific, goal-directed and individualized coagulation therapy. This paragraph offers a short overview considering the most commonly used preparations.

\section{Fibrinogen}

Fibrinogen is one of the key coagulation factors and the substrate to form a clot. In massively bleeding patients, fibrinogen is being consumed at a rate that vastly exceeds its production. Accordingly, this coagulation factor drops disproportionally and becomes critically low early on [47, 48]. Additionally, several in vitro and in vivo studies could show that dilutional coagulopathy can be treated successfully with fibrinogen substitution. The clot strength will improve and the need for transfusions may be lowered. Therefore, it is recommended to replace fibrinogen early and aggressively in massive bleeding (recommended fibrinogen concentration $>1.5-2.0 \mathrm{~g} / \mathrm{L}$, corresponding to MCF levels of greater than $8-10 \mathrm{~mm}$ in fibTEM test of ROTEM) [49].

\section{Prothrombin Complex Concentrates}

Prothrombin complex concentrates (PCC) provide a source of the vitamin $\mathrm{K}$ dependent coagulation factors (FII, FVII,
FIX, FX) together with variable amounts of protein $\mathrm{C} / \mathrm{S}$, antithrombin and heparin, depending on the manufacturer. PCC are approved, both in Europe and the United States, for the perioperative treatment of bleeding patients with deficiencies in the above mentioned clotting factors (e.g., oral anti-coagulation with vitamin $\mathrm{K}$ antagonists) if a quick correction of the deficiency is mandatory. Additionally, there has been emerging evidence that PCC not only help reduce blood product transfusion rates, but also decrease the rate of multiorgan failure and sepsis [50•*]. That has led to PCC being increasingly used off label for perioperative coagulation interventions in severely bleeding patients. A careful risk-benefit analysis must, however, always occur before its use to keep potential thrombo-embolic adverse events low [51].

\section{Activated Recombinant Factor VII}

Activated recombinant factor VII (rFVIIa) leads to a direct thrombin burst, a massive generation of thrombin, and thereby transforming fibrinogen to fibrin. It is approved for the treatment of acquired hemophilia, hereditary factor VII deficiency and thrombasthenia Glanzmann. There is rFVIIahas which has formerly been used off label as "the silver bullet" in otherwise uncontrollable bleeding. Unfortunately, multiple reports have been published on fatal thrombo-embolic adverse events after off label rFVIIa use. Therefore, the EMEA (European Medical agency) explicitly published a warning against the off label use in 2009 [52]. In addition to that, the results of the CONTROL trial in 2010 further questioned the role of rFVIIa. It prospectively randomized 573 trauma patients to receive either placebo or rFVIIa. While a reduced blood product use could be demonstrated, mortality and thromboembolic events did not differ in the two groups [53].

\section{Factor XIII}

Factor XIII is another important clotting factor and plays a central role in clot stability by cross-linking fibrin. Aside from that, FXIII protects fibrinogen and fibrin against an untimely lysis process. Extensive surgery and/or major bleeding may lead to an acquired FXIII deficiency that has been described with increased bleeding diathesis. Therefore, it is recommended to keep FXIII $>60 \%$ during the perioperative period [54].

Fresh Frozen Plasma (FFP) and Platelet Concentrates

Since the transfusion of blood products carries numerous and serious adverse effects, the transfusion of allogeneic blood products must be avoided where possible (restrictive transfusion regimen) and be replaced by alternatives 
whenever possible. It is easily forgotten, that the clinical use of FFP is not supported by any evidence from randomized trials in most clinical circumstances. Furthermore, no studies have taken into consideration that the extent of adverse effects might negate the clinical benefits of treatment with FFP [55]. Up to now, the only legitimate indication for FFP transfusion is a FV deficiency, since specific FV concentrate is not commercially available. A fixed ratio between FFP, red cell concentrates (PRBC) and platelets has been discussed over and over again in the literature [56]. While we completely concur that early and aggressive hemostatic resuscitation is key in massively bleeding patients, we, however, strongly believe that a fixed ratio approach must be abandoned. The fixed ratio concept is solely based on retrospective, hence, biased data ("survivorship bias") and has failed so far to show any benefit in comparison to timedependent data analyses or prospective studies [57•]. The beneficial effect of high plasma to RBC ratios in most of those trials might have been limited by survivorship bias, that is caused by a subgroup of the most severely injured patients, which survive only long enough to be transfused with RBC, but not FFP. Contemporary blood coagulation management should be goal-directed, individualized and specific for each single patient and the given situation [40].

Guidelines for the transfusion of FFP and platelets have been published in the past, however, they are expert-oriented and not evidence-based [58]. Published indications for FFP transfusions are: a severe, diffuse microvascular bleeding, the emergent reversal of oral anti-coagulation (in absence of PCC) and thrombotic thrombocytopenic purpura. Since coagulation factor activity is low, large amounts of FFP have to be transfused to be effective (15 ml FFP/kg BW) [59]. Platelets should be maintained $>50 \times 10^{3} / \mu 1$ in bleeding patients and even raised $>100 \times 10^{3} / \mu 1$ under certain circumstances (e.g., patients with traumatic brain injury, impaired platelet function) [60, 61].

\section{Pharmacological Pro-coagulant Therapy}

\section{Anti-fibrinolytics}

Currently available substances for patients' treatment are $\varepsilon$ aminocaproic acid and tranexamic acid. Both drugs are analogues of lysine and competitively block fibrinolysis. Anti-fibrinolytics can reduce the transfusion requirements in certain situations and have shown to improve the patients' outcome primarily in cardiac surgery, transplant surgery, trauma care, obstetrics and orthopedics. Side effects like seizures as well as distinctive nausea and emesis are to be considered for tranexamic acid. CRASH-2 studied the effect of tranexamic acid in trauma patients specifically. There were 20,211 adult trauma patients in 274 hospitals in 40 countries prospectively randomized to receive either placebo or tranexamic acid. The patients that received tranexamic acid had a significantly lower allcause mortality [10]. In a follow up, the collaborators investigated the data further and found that early treatment was crucial; ideally, tranexamic acid should be given within the first hour of trauma, but certainly not later than $3 \mathrm{~h}[62 \bullet]$. The MATTERs study was able to confirm similar findings in a combat setting [63].

\section{Protamines}

Protamines are alkaline, low molecular weight proteins derived from the testicles of salmon. Protamines are heparin antagonists, binding to the (acid) heparin to form inactive complexes with no anti-coagulant properties. Since the half-life of protamine is shorter than that of heparin, rebound anti-coagulation may occur. Furthermore, protamine administered alone or in excess has weak anticoagulant properties and other undesired side effects.

\section{Desmopressin}

Desmopressin (DDAVP) is being used in certain specific disorders of the primary hemostasis, e.g., the type 1 von Willebrand's syndrome. After a standard dose of DDAVP $(0.3 \mu \mathrm{g} / \mathrm{kg} \mathrm{BW})$, plasma concentration of FVIII and vWF will increase 3-5-fold over 8-12 h. Always consider that DDAVP administration undergoes tachyphylaxis and may increase fibrinolysis.

\section{Conclusions}

The concepts of resuscitation for massively bleeding trauma patients have evolved greatly over the past 15 years. There has been a lot of research focused on the physiology of trauma, hemorrhage and coagulopathy. Combined with the vast experience gained from the armed conflicts, that has led to a better understanding and more advanced concepts on how to care for trauma patients. Traumatic coagulopathy is highly prevalent at an early stage after injury and needs to be aggressively treated. Effective preparation and education of staff is crucial for institutions that care for trauma victims. The care of a given patient needs to be tailored to the patients' needs, utilizing a multitude of diagnostic instruments and therapeutic interventions. Further research will have to investigate more tailored approaches and fine tune treatment algorithms.

\section{Compliance with Ethics Guidelines}

Conflict of Interest Marc P. Steurerhas received honoraria or travel support for consulting and lecturing from TEM Systems, Inc. 
(Durham, NC). Michael T. Ganterhas received honoraria or travel support for consulting and lecturing from CSL Behring $\mathrm{GmbH}$ (Marburg, Germany).

Human and Animal Rights and Informed Consent This article does not contain any studies with human or animal subjects performed by any of the authors.

\section{References}

Papers of particular interest, published recently, have been highlighted as:

- Of importance

•- Of major importance

1. Lozano R, Naghavi M, Foreman K, et al. Global and regional mortality from 235 causes of death for 20 age groups in 1990 and 2010: a systematic analysis for the Global Burden of Disease Study 2010. Lancet. 2012;380:2095-128.

2. Collaborators USBoD. The state of US health, 1990-2010: burden of diseases, injuries, and risk factors. JAMA. 2013;310:591-608.

3. Evans JA, van Wessem KJ, McDougall D, et al. Epidemiology of traumatic deaths: comprehensive population-based assessment. World J Surg. 2010;34:158-63.

4. Sauaia A, Moore FA, Moore EE, et al. Epidemiology of trauma deaths: a reassessment. J Trauma. 1995;38:185-93.

5. Ganter MT, Brohi K, Cohen MJ, et al. Role of the alternative pathway in the early complement activation following major trauma. Shock. 2007;28:29-34.

6. Ganter MT, Pittet JF. New insights into acute coagulopathy in trauma patients. Best Pract Res Clin Anaesthesiol. 2010;24:15-25.

7. • Frith D, Brohi K. The pathophysiology of trauma-induced coagulopathy. Curr Opin Crit Care. 2012;18:631-6. Structured overview on the pathogenesis of traumatic coagulopathy.

8. Davenport R. Pathogenesis of acute traumatic coagulopathy. Transfusion. 2013;53(Suppl 1):23S-7S.

9. - Cohen MJ, Call M, Nelson M et al. Critical role of activated protein $\mathrm{C}$ in early coagulopathy and later organ failure, infection and death in trauma patients. Ann Surg. 2012;255:379-85. Outlines the critical role that protein $C$ and activated protein $C$ play not only for the acute traumatic coagulopathy, but possibly also for other organ dysfunction.

10. Shakur H, Roberts I, Bautista R, et al. Effects of tranexamic acid on death, vascular occlusive events, and blood transfusion in trauma patients with significant haemorrhage (CRASH-2): a randomised, placebo-controlled trial. Lancet. 2010;376:23-32.

11. Howard BM, Daley AT, Cohen MJ. Prohemostatic interventions in trauma: resuscitation-associated coagulopathy, acute traumatic coagulopathy, hemostatic resuscitation, and other hemostatic interventions. Semin Thromb Hemost. 2012;38:250-8.

12. • Spahn DR, Bouillon B, Cerny V et al. Management of bleeding and coagulopathy following major trauma: an updated European guideline. Crit Care. 2013;17:R76. Comprehensive, evidencebased recommendation for the acute management of bleeding trauma patients.

13. Attar S, Boyd D, Layne E, et al. Alterations in coagulation and fibrinolytic mechanisms in acute trauma. J Trauma. 1969;9:939-65.

14. McNamara JJ, Burran EL, Stremple JF, et al. Coagulopathy after major combat injury: occurrence, management, and pathophysiology. Ann Surg. 1972;176:243-6.

15. MacLeod JB, Lynn M, McKenney MG, et al. Early coagulopathy predicts mortality in trauma. J Trauma. 2003;55:39-44.
16. Carroll RC, Craft RM, Langdon RJ, et al. Early evaluation of acute traumatic coagulopathy by thrombelastography. Transl Res. 2009;154:34-9.

17. Floccard B, Rugeri L, Faure A, et al. Early coagulopathy in trauma patients: an on-scene and hospital admission study. Injury. 2012;43:26-32.

18. Brohi K, Cohen MJ, Ganter MT, et al. Acute coagulopathy of trauma: hypoperfusion induces systemic anticoagulation and hyperfibrinolysis. J Trauma. 2008;64:1211-7 discussion 7.

19. Frith D, Goslings JC, Gaarder C, et al. Definition and drivers of acute traumatic coagulopathy: clinical and experimental investigations. J Thromb Haemost. 2010;8:1919-25.

20. Wafaisade A, Wutzler S, Lefering $R$, et al. Drivers of acute coagulopathy after severe trauma: a multivariate analysis of 1987 patients. Emerg Med J. 2010;27:934-9.

21. Hubetamann B, Lefering R, Taeger $G$, et al. Influence of prehospital fluid resuscitation on patients with multiple injuries in hemorrhagic shock in patients from the DGU trauma registry. J Emerg Trauma Shock. 2011;4:465-71.

22. Raza I, Davenport R, Rourke C, et al. The incidence and magnitude of fibrinolytic activation in trauma patients. $\mathrm{J}$ Thromb Haemost. 2013;11:307-14.

23. Solomon C, Traintinger S, Ziegler B, et al. Platelet function following trauma: a multiple electrode aggregometry study. Thromb Haemost. 2011;106:322-30.

24. Holcomb JB, Zarzabal LA, Michalek JE, et al. Increased platelet: $\mathrm{RBC}$ ratios are associated with improved survival after massive transfusion. J Trauma. 2011;71:S318-28.

25. Ostrowski SR, Johansson PI. Endothelial glycocalyx degradation induces endogenous heparinization in patients with severe injury and early traumatic coagulopathy. J Trauma Acute Care Surg. 2012;73:60-6.

26. Pham HP, Shaz BH. Update on massive transfusion. Br J Anaesth. 2013;111(Suppl 1):i71-82.

27. Cotton BA, Au BK, Nunez TC, et al. Predefined massive transfusion protocols are associated with a reduction in organ failure and postinjury complications. J Trauma. 2009;66:41-8 discussion 8-9.

28. Jansen JO, Thomas R, Loudon MA, et al. Damage control resuscitation for patients with major trauma. BMJ. 2009;338:b1778.

29. Dutton RP. Haemostatic resuscitation. Br J Anaesth. 2012; 109(Suppl 1):i39-46.

30. Napolitano LM, Kurek S, Luchette FA, et al. Clinical practice guideline: red blood cell transfusion in adult trauma and critical care. J Trauma. 2009;67:1439-42.

31. Koscielny J, Ziemer S, Radtke H, et al. A practical concept for preoperative identification of patients with impaired primary hemostasis. Clin Appl Thromb Hemost. 2004;10:195-204.

32. Pfanner G, Koscielny J, Pernerstorfer T, et al. Preoperative evaluation of the bleeding history: recommendations of the working group on perioperative coagulation of the Austrian Society for Anaesthesia, Resuscitation and Intensive Care. Anaesthesist. 2007;56:604-11.

33. Ganter MT, Hofer CK. Coagulation monitoring: current techniques and clinical use of viscoelastic point-of-care coagulation devices. Anesth Analg. 2008;106:1366-75.

34. Perry DJ, Fitzmaurice DA, Kitchen S, et al. Point-of-care testing in haemostasis. Br J Haematol. 2010;150:501-14.

35. Hofer CK, Zollinger A, Ganter MT. Perioperative assessment of platelet function in patients under antiplatelet therapy. Expert Rev Med Devices. 2010;7:625-37.

36. Michelson AD. Methods for the measurement of platelet function. Am J Cardiol. 2009;103:20A-6A.

37. - Stensballe J, Ostrowski SR, Johansson PI. Viscoelastic guidance of resuscitation. Curr Opin Anaesthesiol. 2014;27: 212-8. Concise review on thrombelastometry and its integration in resuscitation. 
38. Kozek-Langenecker SA, Afshari A, Albaladejo P, et al. Management of severe perioperative bleeding: guidelines from the European Society of Anaesthesiology. Eur J Anaesthesiol. 2013;30:270-382.

39. Ganter MT, Spahn DR. Active, personalized, and balanced coagulation management saves lives in patients with massive bleeding. Anesthesiology. 2010;113:1016-8.

40. Spahn DR, Ganter MT. Towards early individual goal-directed coagulation management in trauma patients. $\mathrm{Br} \mathrm{J}$ Anaesth. 2010;105:103-5.

41. Levi M, Fries D, Gombotz H, et al. Prevention and treatment of coagulopathy in patients receiving massive transfusions. Vox Sang. 2011;101:154-74.

42. Theusinger OM, Spahn DR, Ganter MT. Transfusion in trauma: why and how should we change our current practice? Curr Opin Anaesthesiol. 2009;22:305-12.

43. - Schochl H, Schlimp CJ. Trauma bleeding management: the concept of goal-directed primary care. Anesth Analg. 2013. Good comparison between ratio-driven vs. point-of-care guided coagulation management in traumatic hemorrhage.

44. Darlington DN, Kheirabadi BS, Delgado AV, et al. Coagulation changes to systemic acidosis and bicarbonate correction in swine. J Trauma. 2011;71:1271-7.

45. Lier H, Krep H, Schroeder S, et al. Preconditions of hemostasis in trauma: a review - the influence of acidosis, hypocalcemia, anemia, and hypothermia on functional hemostasis in trauma. J Trauma. 2008;65:951-60.

46. Meng ZH, Wolberg AS, Monroe DM 3rd, et al. The effect of temperature and $\mathrm{pH}$ on the activity of factor VIIa: implications for the efficacy of high-dose factor VIIa in hypothermic and acidotic patients. J Trauma. 2003;55:886-91.

47. Hiippala ST, Myllyla GJ, Vahtera EM. Hemostatic factors and replacement of major blood loss with plasma-poor red cell concentrates. Anesth Analg. 1995;81:360-5.

48. Schlimp CJ, Voelckel W, Inaba K, et al. Estimation of plasma fibrinogen levels based on hemoglobin, base excess and Injury Severity Score upon emergency room admission. Crit Care. 2013;17:R137.

49. Rossaint R, Bouillon B, Cerny V, et al. Management of bleeding following major trauma: an updated European guideline. Crit Care. 2010;14:R52.

50. • Innerhofer P, Westermann I, Tauber $\mathrm{H}$ et al. The exclusive use of coagulation factor concentrates enables reversal of coagulopathy and decreases transfusion rates in patients with major blunt trauma.
Injury. 2013;44:209-16. The first prospective trial comparing a coagulation factor-only approach with a combined FFP plus coagulation factors regime.

51. Samama CM. Prothrombin complex concentrates: a brief review. Eur J Anaesthesiol. 2008;25:784-9.

52. Levi M, Levy JH, Andersen HF, et al. Safety of recombinant activated factor VII in randomized clinical trials. N Engl J Med. 2010;363:1791-800.

53. Hauser CJ, Boffard K, Dutton R, et al. Results of the CONTROL trial: efficacy and safety of recombinant activated Factor VII in the management of refractory traumatic hemorrhage. J Trauma. 2010;69:489-500.

54. Korte WF. F. XIII in perioperative coagulation management. Best Pract Res Clin Anaesthesiol. 2010;24:85-93.

55. Yang L, Stanworth S, Hopewell S, et al. Is fresh-frozen plasma clinically effective? an update of a systematic review of randomized controlled trials. Transfusion. 2012;52:1673-86 quiz.

56. Johansson PI. Hemostatic strategies for minimizing mortality in surgery with major blood loss. Curr Opin Hematol. 2009; 16:509-14.

57. - Halmin M, Bostrom F, Brattstrom O et al. Effect of plasma-toRBC ratios in trauma patients: a cohort study with time-dependent data*. Crit Care Med. 2013;41:1905-14. Evaluation of the association of plasma-to-PRB ratio and risk of death in trauma patients under consideration of the survival bias.

58. Schiffer CA. What to do if there is no evidence? the issue of surgical procedures in patients with thrombocytopenia. Transfusion. 2011;51:2262-4.

59. Kozek-Langenecker S, Sorensen B, Hess JR, et al. Clinical effectiveness of fresh frozen plasma compared with fibrinogen concentrate: a systematic review. Crit Care. 2011;15:R239.

60. Kor DJ, Stubbs JR, Gajic O. Perioperative coagulation management-fresh frozen plasma. Best Pract Res Clin Anaesthesiol. 2010;24:51-64.

61. Spiess BD. Platelet transfusions: the science behind safety, risks and appropriate applications. Best Pract Res Clin Anaesthesiol. 2010;24:65-83.

62. • Roberts I, Shakur H, Ker K et al. Antifibrinolytic drugs for acute traumatic injury. Cochrane Database Syst Rev. 2012;12:CD004896. Collection of a large body of evidence for tranexamic acid, and the need to apply it early.

63. Morrison JJ, Dubose JJ, Rasmussen TE, et al. Military application of tranexamic acid in trauma emergency resuscitation (MATTERs) Study. Arch Surg. 2012;147:113-9. 